\title{
Creación de programas para la educación en valores en la etapa preescolar: Incumbencia del terapeuta ocupacional que trabaja en educación
}

\section{Creation of programs for the education in values in the preschooler stage: A objective of the Occupational Therapist to work in education}

Este artículo ofrece a maestros y terapeutas ocupacionales un espacio de reflexión sobre las bases teóricas, lineamientos educativos y desarrollo de programas para iniciar al niño preescolar en la formación en valores humanos y posturas de convivencia social.

Resalta la incumbencia del terapeuta ocupacional en autores como Trujillo, Schwarts y Engquist, quienes han resaltado la importancia de la acción transformadora del terapeuta ocupacional en la comunidad. Utiliza a Hopkins, Schwarts, Chen, Kielhofner y De Las Heras, para ubicar el área del desempeño ocupacional que influye sobre la formación en valores.

MARTHA María TobóN

Terapeuta Ocupacional

Universidad Nacional de Colombia

Especialista en Docencia Universitaria

Colegio Mayor de Nuestra Señora del Rosario

E-mail: awadtobon@hotmail.com

Por un mañana

Carrera 68 Transversal 62 No 174-51

San José de Bavaria

Bogotá, D. C. - Colombia
Define los valores y su relación con los derechos y deberes de los niños, su proceso de construcción y la importancia de enseñarlos tempranamente. Muestra el currículo para su construcción de acuerdo con los lineamientos colombianos para educación preescolar. Hace énfasis en el papel del maestro y del terapeuta ocupacional.

Ejemplifica la propuesta conceptual con el programa de desarrollo emocional del "Jardín Infantil Por un Mañana" en Bogotá, Colombia. 
Valores, derechos y deberes de los niños, voluntad y claves valores éticos, desempeño ocupacional y valores humanos, valores humanos y educación.

The purpose of this article is to open a space of thinking on theorical basis of teaching values and social behavior to preschool children. It concern to occupational therapist who work on building values. It emphasizes their role when teaches values.

It highlights the important role the O.T. plays about building of values in direct connection with the community engaged in communal life.

Defines values and their relationship with children rights and their obligations. It talks about how to promote values and their importance to do this during the preschool years.

The article also shows how the Colombian preschool curriculum is design to teach values according to our culture.

It mention s some authors who have written about O.T. importance in this area. They are Trujillo, Schwarts \& Engquist,,Hopkins, Chen, Kielhofner or De Las Heras.

Lastly, talks about the development and implementation of an emotional intelligence program at "Por un Mañana" preschool in Bogotá, Colombia.

\section{$K \in y$ words}

Social values, human rights and obligations, volition and values, occupational performance and values, education and values. 
"Mantener una relación ética con los otros es estar siempre dispuesto a concederles la palabra y a poner en palabras lo que exigimos de ellos, lo que ofrecemos o lo que les rechazamos".

El objetivo de este artículo es ofrecer a los maestros y terapeutas ocupacionales que se desempeñan en el campo educativo, un espacio de reflexión sobre las bases teóricas, los lineamientos educativos y el desarrollo de los programas para iniciar al niño preescolar en la formación en valores éticos y posturas de convivencia social, resaltando su papel como educadores.

Primero se definirá lo que son los valores humanos y su relación con los derechos y deberes de los niños. Después se ubicará el área del desempeño ocupacional que influye sobre la formación en valores. Luego se hablará sobre el proceso de construcción de valores. A continuación se resaltará la importancia de la formación en valores durante la etapa preescolar. Posteriormente se mostrará en qué consiste el currículo preescolar para la construcción de esos valores. Luego se mostrará el papel del maestro, del terapeuta ocupacional y del con- texto escolar en la educación en valores. Finalmente se utilizará el programa de formación de valores del Jardín Infantil Por un Mañana, como un ejemplo de la implementación de la propuesta teórica aqui planteada.

La base teórica de este artículo en formación de valores se apoya en autores como Lev Vigotsky, Luis Carlos Restrepo, Martha Rodriguez, Agustín Nieto Caballero, Helena Ortiz de Maschwitz, y Manuel Iceta, entre otros. La incumbencia del terapeuta Ocupacional en la formación de los valores en los niños se sustenta en autores como Helen Hopkins, Barquer Schwarts, Jen Suh Chen, Gary Kielhofner, Carmen De Las Heras, Alicia Trujillo, Schwarts, y Engquist, quienes han resaltado la relación de los valores con el desempeño ocupacional y la importancia de la actuación del terapeuta ocupacional en relación directa con la comunidad, durante su ejercicio profesional.

\section{Los valores humanos y su relación con los derechos y deberes de los niños}

Según los lineamientos curriculares del preescolar, los valores son los principios que orientan la actitud ética y moral de las personas para relacionarse con el entorno y tomar decisiones sobre como vivir (Ministerio de Educación Nacional, 1998). Con base en éstos el hombre clasifica sus actuaciones en una escala de prioridades éticas que se convierten en la medida 
con la cual califica, aprueba o desaprueba las acciones propias y las de los demás.

La construcción de la escala de valores se inicia desde la niñez gracias a la interacción con el entorno social el cual ofrece un referente externo con el cual contrastar las propias ideas sobre los principios que regirán la vida (Chen, Kielhofner y De las Heras, 1996). Por lo tanto los valores que el individuo desarrolla durante sus primeros años son definitivos para la adopción de la postura ética que le permitirá aceptar los deberes con la sociedad, respetar los derechos de los demás y hacer valer los propios, entre otras acciones.
Por otra parte, la práctica y respeto de los derechos y deberes aceptados por un contexto social determinado, favorece el aprendizaje y entendimiento de los valores de acuerdo con la idiosincrasia que mueve sus acciones (Rodríguez, 1997). O sea que es vital tener en cuenta cuales son los deberes y derechos que se practican en cada grupo social pues éstos llevan al niño a desarrollar ciertos valores humanos que le identifican con su comunidad. En Colombia se tienen en cuenta los deberes y derechos propuestos en convención sobre los derechos del niño (1). El cuadro No. 1 define los derechos, los deberes y los valores y ejemplifica su relación.

\begin{tabular}{|c|c|c|}
\hline $\begin{array}{l}\text { DERECHO } \\
\text { LO QUE NO SE PUEDE NEGAR. } \\
\text { LO QUE ES JUSTO CONMIGO. } \\
\text { LO QUE PUEDO HACER POR MÍ. }\end{array}$ & $\begin{array}{l}\text { DEBER QUE CONLLEVA } \\
\text { LO QUE NO PUEDO NEGARA LOS } \\
\text { DEMÁS. LO QUE ES JUSTO CON } \\
\text { OTROS. LO QUE TENGO QUE HA- } \\
\text { CER PARA VIVIR EN COMUNIDAD. }\end{array}$ & $\begin{array}{l}\text { VALOR QUE LO PROMUEVE } \\
\text { LOS PRINCIPIOS PARA CONDU- } \\
\text { CIR MIS ACCIONES. LO QUE ES } \\
\text { PRIORITARIO PARA DECIDIR } \\
\text { COMOACTUAR. }\end{array}$ \\
\hline $\begin{array}{l}\text { Derecho a un nombre y una na- } \\
\text { cionalidad }\end{array}$ & $\begin{array}{l}\text { Respetar a su familia. } \\
\text { Amar y respetar a su pais } \\
\text { Hablar bien de su familia y su país }\end{array}$ & $\begin{array}{l}\text { - Fidelidad } \\
\text { - Respeto } \\
\text { - Responsabilidad }\end{array}$ \\
\hline Derecho a la vida & $\begin{array}{l}\text { No jugar a la guerra. } \\
\text { No matar ningún ser vivo } \\
\text { Quererse y valorarse como ser único } \\
\text { No consumir drogas }\end{array}$ & $\begin{array}{l}\text { - Respeto } \\
\text { - Justicia } \\
\text { - Responsabilidad }\end{array}$ \\
\hline Derecho a la educación & $\begin{array}{l}\text { Asistir a la escuela cumplidamente. } \\
\text { Aprovechar lo que me enseñan. } \\
\text { Estudiar } \\
\text { Preferir el estudio antes que } \\
\text { aceptar trabajos riesgosos. }\end{array}$ & $\begin{array}{l}\text { - Responsabilidad } \\
\text { - Respeto } \\
\text { - Honestidad } \\
\text { - Solidaridad } \\
\text { - Tolerancia }\end{array}$ \\
\hline Derecho al amor y a la familia & $\begin{array}{l}\text { Amar a los padres. } \\
\text { Acompañarlos. } \\
\text { No olvidar las tradiciones de los } \\
\text { abuelos. } \\
\text { Dar buen trato y amor a otros. } \\
\text { Ayudar en los oficios de la casa. }\end{array}$ & $\begin{array}{l}\text { - Fidelidad } \\
\text { - Reconciliación } \\
\text { - Tolerancia } \\
\text { - Respeto } \\
\text { - Responsabilidad } \\
\text { - Justicia }\end{array}$ \\
\hline Derecho al ambiente sano & $\begin{array}{l}\text { Cuidar las plantas. } \\
\text { Defender la naturaleza. } \\
\text { Defender los animales. } \\
\text { Disfrutar y cuidar el agua. }\end{array}$ & $\begin{array}{l}\text { - Respeto } \\
\text { - Responsabilidad }\end{array}$ \\
\hline
\end{tabular}




\begin{tabular}{|c|c|c|}
\hline Derecho a la diferencia & $\begin{array}{l}\text { Respetarnos. } \\
\text { Aceptar que existen opiniones } \\
\text { diferentes. } \\
\text { Reconocer el valor de la dife- } \\
\text { rencia. }\end{array}$ & $\begin{array}{l}\text { - Tolerancia } \\
\text { - Respeto } \\
\text { - Sinceridad } \\
\text { - Justicia }\end{array}$ \\
\hline Derecho al cuidado especial & $\begin{array}{l}\text { Cuidar al minusválido } \\
\text { Apoyar al débil. } \\
\text { Atender primero al mas débil. } \\
\text { Buscar ayuda si estoy en proble- } \\
\text { mas }\end{array}$ & $\begin{array}{l}\text { - Reconocer la diferencia } \\
\text { - Justicia } \\
\text { - Responsabilidad } \\
\text { - Solidaridad } \\
\text { - Tolerancia }\end{array}$ \\
\hline $\begin{array}{l}\text { Derecho a no trabajar antes de } \\
\text { la edad permitida }\end{array}$ & $\begin{array}{l}\text { Escoger el estudio antes que los } \\
\text { trabajos riesgosos. } \\
\text { Aprovechar lo que recibimos en } \\
\text { casa. } \\
\text { Ayudar en los oficios de la casa. }\end{array}$ & $\begin{array}{l}\text { - Justicia } \\
\text { - Solidaridad } \\
\text { - Respeto } \\
\text { - Colaboración }\end{array}$ \\
\hline $\begin{array}{l}\text { Derecho a la libertad de expre- } \\
\text { sión }\end{array}$ & $\begin{array}{l}\text { Escuchar a los demás. } \\
\text { No ofender. } \\
\text { Comprendernos. }\end{array}$ & $\begin{array}{l}\text { - Tolerancia } \\
\text { - Respeto } \\
\text { - Honestidad } \\
\text { - Sinceridad }\end{array}$ \\
\hline Derecho a ser los primeros & $\begin{array}{l}\text { Atender primero al mas pequeño. } \\
\text { Ser solidario. } \\
\text { Hacer grupos de apoyo durante } \\
\text { los desastres. }\end{array}$ & $\begin{array}{l}\text { - Solidaridad } \\
\text { - Respeto } \\
\text { - Justicia }\end{array}$ \\
\hline Derecho al buen trato & $\begin{array}{l}\text { Dar buen trato y amor a otros. } \\
\text { No ofender. } \\
\text { Respetarnos. } \\
\text { Contarle a tu familia si alguien } \\
\text { trata de abusar de tu cuerpo. } \\
\text { No dejarse convencer para co- } \\
\text { meter delitos. }\end{array}$ & $\begin{array}{l}\text { - Honestidad } \\
\text { - Justicia } \\
\text { - Reconciliación } \\
\text { - Solidaridad }\end{array}$ \\
\hline Derecho a la paz & $\begin{array}{l}\text { Ser constructor de paz. } \\
\text { No jugar a la guerra. } \\
\text { Respetar a todo ser vivo. }\end{array}$ & $\begin{array}{l}\text { - Reconciliación } \\
\text { - Solidaridad } \\
\text { - Respeto } \\
\text { - Justicia }\end{array}$ \\
\hline
\end{tabular}

Fuente: Comisión internacional de juristas (1); Rodriguez (2). Movimiento por la paz de Colombia(3)

Cuadro No. 1. Derechos, deberes y valores humanos.

Esta relación derecho-deber-valor es tan estrecha que se puede decir que su enseñanza debe verse como una sola construcción de conocimiento del sentido ético en los niños y niñas.
Para resumir se puede decir que el conocimiento de los derechos, deberes y el desarrollo de los valores humanos son los lineamientos que determinan la actitud ética y moral de las personas. De ésta dependerá su postura ante la vida, sus actitudes y sus decisiones. 


\section{Desempeño ocupacional y formación en valores}

La escala de valores se construye en la esfera espiritual y ética la cual es uno de los componentes del ser humano: cuerpo, mente y espíritu (Kielhofner 1992, Engquist y colaboradores, 1997; Howard y Howard, 1997). Esta esfera se puede ubicar dentro del subsistema de la voluntad planteado por Kielhofner en el Modelo de la Ocupación Humana. La voluntad favorece:

- La disposición emocional y cognitiva con que se mira el mundo.

- La toma de decisiones para dar sentido al uso del tiempo e involucrarse en ciertas ocupaciones.

- La elección de valores éticos que se consideran importantes al escoger las ocupaciones durante la vida.

- La formación del autoconcepto, el cual dirige las actuaciones personales.

La voluntad por lo tanto, influye de manera vital en el adecuado desempeño ético del individuo ya que determina la integridad personal, los valores humanos que se aceptan y respe$\tan$ y las decisiones que rigen las actuaciones comunitarias.

Esta elección de los valores éticos a respetar también determina de mane- ra significativa la forma en que las personas desempeñan sus ocupaciones, los juicios que hacen sobre sus actos y los de los demás así como las actuaciones que consideran vitales para su comunidad (Hopkins, 1998).

A ésta manera de actuar se le denomina sentido ético (Engquist y colaboradores, 1997). Éste determina las decisiones del individuo con relación a:

- Las actividades que está dispuesto a realizar

- La identificación de sueños y metas

- La escogencia de reglas de vida

- La manera se ser y hacer las cosas

- O el sentido ético para participar de las diversas ocupaciones.

Para resumir se puede decir que en el sistema de la voluntad, el individuo crea una serie de normas de conducta para decidir lo que es bueno o lo que está correcto. Estas normas de comportamiento están basadas en valores éticos los cuales respeta y está dispuesto a cumplir. Además cada una de las elecciones ocupacionales y de las actuaciones humanas está determinada por esas creencias y valores de la esfera espiritual $y$ ética. 


\section{Proceso de la construcción de valores en los niños}

El niño tiene unas características de pensamiento y desarrollo emocional que le permiten empezar a crear y aceptar principios y valores humanos desde la etapa preescolar (Vigotski, 1981; Labinowicz, 1982; Restrepo de Franco y colaboradoras,1992; Rodríguez, 1997).

La razón radica en que el niño tiene funciones simbólicas a partir del año y medio tales como la memoria evocativa, la inteligencia representativa, la imagen mental o la imitación diferida lo cual le permite construir imágenes mentales de las experiencias vividas, recordarlas y proyectarlas en situaciones posteriores (Labinowicz, 1982; Vigotski, 1981Sauri Suárez, 2000). Estas capacidades son las que facilitan al niño el entendimiento y la aceptación de las acciones propias y las de los demás, para desarrollar a partir de ellas juicios valorativos.

Otra razón que le permite al niño crear valores es la maneara de pensar de los niños en edad preescolar: están en la etapa Preoperacional del pensamiento, la cual se caracteriza por la irreversibilidad (incapacidad de invertir mentalmente una acción física para regresar un objeto a su estado original) y por el egocentrismo (incapacidad de tener en cuenta el pensamiento del otro). Estas características de pensamiento llevan al niño a iniciar la creación de valores a partir de tres supuestos:

- Creer que los demás piensan como él y que entienden como actúa sin necesidad de explicarles el por qué de sus acciones.

- Juzgar las situaciones por su apariencia exterior sin analizarlas ni tener en cuenta las razones de los otros.

- Tener un primer acercamiento a la escala de valores a partir de intereses concretos, necesidades personales y relaciones muy cercanas.

La última razón que favorece el desarrollo de valores en un preescolar es el desarrollo evolutivo del afecto y el pensamiento infantil, lo cual tiene gran importancia en la creación de los sistemas valorativos del niño (Vigotski, 1981; Pinto, 1990; Restrepo de Franco y colaboradoras, 1992). Este proceso se logra gracias al desarrollo progresivo de la escala valorativa del niño, quien va cambiando sus opciones en la medida que se enfrenta a las situaciones de la vida diaria, las cuales le exigen replantear sus ideas acerca de la jerarquía de los valores.

Finalmente las interacciones sociales tienen un papel vital en el desarrollo valorativo de los niños ya que son generadoras de experiencias significativas para que el niño comprenda y acepte los principios y valores sociales de manera gradual (Vigotski, 1981; Iceta, 1985; Restrepo, 1992; Amar y Amar, 2000). En otras palabras el ambiente ofrece la imposición de las normas de los demás (padres, maestros y otros adultos), la creación de reglas propias que en niño impone a los demás y la creación colectiva de límites 
y normas a partir de la cooperación y las acciones democráticas.

El desarrollo de la capacidad valorativa se produce siguiendo tres etapas: la creación de valores específicos, el desarrollo de valores particulares y la construcción de valores generales:

- El desarrollo de los valores específicos corresponde a la etapa preescolar se caracteriza por su estrecha relación con las emociones (intensidad, fuerza, variabilidad y poca duración). También tiene que ver con la influencia de las relaciones con los demás para la aceptación de los valores. Por ejemplo, la amistad entre dos niños puede depender de que uno de ellos traiga un juguete llamativo al jardín y no del concepto de amistad en si.

- El desarrollo de valores particulares depende de la situación ya que se ha visto que los niños menores de cinco años muestran la capacidad de experimentar valores como la solidaridad hacia un compañero que sufre o hacia una mascota. El ejercicio de éste principio puede ser transitorio ya que el niño lo hace de acuerdo con el momento, por esta razón puede ser difícil para el maestro encontrar esa solidaridad en toda ocasión y en todos los ni- ños. Además es frecuente ver la inestabilidad o pobre estructuración de los valores ya que el niño preescolar puede pasar de un sentimiento valorativo al contrario en pocos minutos.

- En la creación de los valores generales se resalta el importante papel de los demás para la creación de valores del niño preescolar ya que éste imita constantemente los comportamientos de otros niños y acepta la figura de autoridad de los adultos como inicio del valor del respeto.

Es importante anotar que en esta época de la vida se observa gran variabilidad para recordar las normas de convivencia. Esto explica por qué los niños a veces recuerdan las normas $y$ las aplican y otras veces no lo hacen. Lo anterior refuerza la teoria de que la construcción de valores en los primeros años es un proceso que varia de acuerdo con el desarrollo del pensamiento y es moldeado por la interacción social.

El desarrollo de principios y valores sigue un proceso que puede extenderse hasta la vida adulta ya que es necesario que el individuo llegue al pensamiento formal para comprender, respetar, aceptar y practicar principios y valores éticos.

\section{La formación en valores durante la etapa preescolar}

El objetivo de la educación, desde los primeros años, es dar a las personas estrategias para dirigir su vida, contribuir con acciones en bien de los de- 
más y ser partícipes de una sociedad responsable y justa (4). Por lo tanto el espacio educativo debe ofrecer al niño oportunidades para construir sus valores a partir de las experiencias e interacciones con los demás.

Para lograr ese proceso la actual estructura curricular de los preescolares colombianos se basa en cuatro espacios: Aprender a conocerse, aprender a hacer, aprender a vivir juntos y aprender a ser persona. Estos espacios, fundamentales para la construcción de conocimiento, se basan en la propuesta que Jacques Delors presentó ante la Comisión de Educación de la Unesco en 1997 (Ministerio de Educación Nacional, 1998). Cada espacio de conocimiento plantea estrategias para favorecer el desarrollo de valores:

Aprender a conocer permite al niño comprender el mundo que le rodea y conocerse a sí mismo. A partir de esto puede empezar a plantear principios de respeto hacia si mismo y hacia los demás, valorar la vida como un don maravilloso y actuar de manera digna con su entorno.

Aprender a hacer tiene que ver con el desarrollo de las habilidades para enfrentar las situaciones cotidianas, resolver los problemas y formar parte de un grupo. Esta interacción permite al niño empezar a desarrollar la tolerancia, la solidaridad, la cooperación y el respeto. Por otra parte, el proceso de hacer exige el establecimiento de relaciones afectivas significativas y le da al niño argumentos para iniciarse en la formación como un ciudadano con deberes y derechos.
Aprender a vivir juntos es el camino para saber compartir con los demás a partir del conocimiento del otro y el sentido de grupo. El niño descubre la existencia del otro cuando ingresa al preescolar y tiene que interactuar con compañeros, maestros y personal del jardín. Con los demás el niño comienza su aprendizaje sobre la tolerancia, la diversidad y el respeto a la diferencia. Cuando el niño experimenta la vida en comunidad, puede percibir por primera vez la construcción de objetivos comunes que le enseñan sobre el valor de la cooperación. Por otra parte el preescolar comienza a descubrir y entender la importancia de conocer y hacer respetar sus derechos, asi como los deberes que estos conllevan.

Aprender a ser es el producto de los anteriores espacios educativos, pues un individuo con libertad de pensamiento y acción es capaz de decidir su camino. En el preescolar el niño inicia ese proceso de ser persona cuando aprende a conocerse a si mismo y reconoce la existencia del otro y del grupo. Esto le permite iniciar la construcción de valores que se consolidarán en su vida adulta. Así estará en capacidad de comprometerse como persona, miembro de una familia y parte productiva de su comunidad. Cuando el niño preescolar está aprendiendo a ser, puede empezar a experimentar valores como la justicia, la honestidad y la solidaridad.

En sintesis, aprender a conocer, aprender a hacer, aprender a vivir juntos y aprender a ser persona se constituyen en los espacios educativos con que cuentan los currículos preesco- 
lares para iniciar al niño en la formación en valores. Estos espacios de formación personal sembrarán la semilla para construir valores éticos que le permitan al niño ser una persona libre y capaz de dar a los demás lo mejor de sí buscando primero el bien común.

\section{El currículo preescolar para la construcción de valores}

Para iniciar al niño en edad preescolar en la formación en valores es necesario utilizar estrategias como la experimentación, la reflexión y la interacción con el mundo que le rodea. Estas estrategias se pueden utilizar mientras se adquieren los conocimientos en todas las dimensiones de la vida (Ministerio de educación Nacional, 1998). A continuación se mostrará la importancia de cada dimensión del desarrollo en la construcción de valores y se mostrará cómo la totalidad de las actividades y situaciones que se dan en un preescolar favorecen la formación de valores.

Dimensión socioafectiva: El desarrollo de la inteligencia emocional es determinante vital de la personalidad, la autoestima y la autonomía. A partir de estas habilidades el niño puede relacionarse con los demás y mostrar su manera de ser, de sentir, de expresar emociones y sentimientos y de juzgar las acciones propias y de los demás. Esta dimensión se desarrolla a través de las clases de desarrollo emocional $y$ ética.

Dimensión corporal: El desarrollo sensoriomotor permitirá al niño expresar su afectividad y su manera de pen- sar a través del movimiento. Con acciones corporales el niño estará en capacidad de comunicar al entorno sus deseos, sentimientos y necesidades. Desde ésta dimensión es que se produce la identificación como persona individual que se quiere a sí misma y se relaciona con los demás a través del contacto físico. Esta dimensión se desarrolla a través de las clases de educación física, expresión corporal o teatro.

Dimensión cognitiva: La capacidad de pensar y formular hipótesis sobre las situaciones de la vida es determinante en las capacidades que tiene el niño para relacionarse, actuar e interpretar la realidad. Gracias al desarrollo cognitivo el niño puede hacer la simbolización de los principios y valores que construye a partir de las relaciones con los demás. También interioriza las acciones para resolver los conflictos de la vida cotidiana y adquiere herramientas de conocimiento para resolverlos. Esta dimensión se trabaja con clases como desarrollo de proyectos cognitivos, democracia, ciencias, lectura, muñequero, etcétera.

Dimensión comunicativa: La utilización del lenguaje se convierte en el 
instrumento para expresar los valores que se están formando. Hablando el niño puede contar lo que piensa sobre las situaciones, establecer relaciones, expresar emociones y defender sus opiniones. Esta dimensión se desarrolla en todas las clases y actividades del jardín.

Dimensión estética: La expresión artística favorece la autoexpresión de sentimientos, ideas y convicciones a través del arte. Cuando el niño expresa su sensibilidad artística puede plasmar valores con relación a sí mismo como ser humano y a los demás. Esta dimensión se facilita con las clases de arte, música, teatro, títeres, trabajo manual, etcétera.

Dimensión espiritual: Se refiere a la posibilidad de trascender a partir del conjunto de valores y principios morales. Esta dimensión tiene una gran in- fluencia de las creencias familiares y culturales. Esta dimensión se desarrolla en las clases de catequesis y religión.

Dimensión ética: Tiene que ver con la forma en que el niño va aprendiendo a dirigir su vida para relacionarse con el entorno. De esta dimensión depende la construcción de principios y valores que marcarán el papel que el niño asumirá en su comunidad. También será determinante de la actitud autónoma del niño. Esta dimensión se desarrolla en las clases de desarrollo emocional, democracia, sexualidad, catequesis, etcétera.

En resumen, la formación en valores se favorece cuando se estimula el desarrollo integral de todas las dimensiones a partir de las clases y actividades que conforman el currículo preescolar.

\section{El papel del contexto escolar, del maestro y del terapeuta ocupacional en los programas de educación en valores}

El contexto escolar en la educación en valores: El jardín infantil es el primer espacio social en que el niño tiene que desempeñarse de manera autónoma. Por lo tanto es el lugar donde comienza la construcción de valores y principios para la convivencia tales como la solidaridad, la tolerancia, la reconciliación y la justicia (Rodriguez, 1997).
En el jardín el niño también aprende rutinas que lo inician en la comprensión de sus derechos y deberes como miembro de la sociedad. Con la participación en las diversas actividades descubre que puede organizarse e interactuar con los compañeros en diferentes espacios, los cuales tienen reglas especiales, las cuales le permiten disfrutar con mayor facilidad de las actividades propuestas. 
Cuando el niño ingresa al preescolar se enfrenta a normas y reglas nuevas que favorecen el desarrollo de habilidades de concertación, reconciliación y respeto. Además aprende a crear normas que comparte con sus compañeros y respeta con gusto ya que han nacido de su propio deseo de organizar el ambiente que le rodea.

Además en el jardín infantil el niño también tiene que responder por sus objetos personales, tareas y razones para casa o colegio, aprendiendo así sobre la responsabilidad y el compromiso.

En el preescolar el niño también tiene que cuidar los espacios comunes y compartir los objetos. Esto le permite descubrir la importancia del bien común, la igualdad derechos y deberes y la existencia de reglas para todos. Esta será la base del comportamiento cívico.

Finalmente las actividades preescolares basadas en la lúdica, la expresión personal, el juego y la exploración son el espacio perfecto para que el niño se sienta a gusto de practicar sus primeros acercamientos a los principios éticos, valores humanos, derechos $y$ deberes.

\section{El papel del maestro y del terapeu-} ta ocupacional en la educación en valores: La responsabilidad de los maestros y de los terapeutas que trabajan en el área educativa en la tarea de iniciar a los niños en la formación de valores es muy grande ya que ellos son el modelo que los niños miran constantemente (Young, 1910, Dewey, 1929, Schwarts,1992). Por éste moti- vo es de vital importancia que tengan presente la necesidad de estar trabajando constantemente con base en actitudes éticas que su alumno pueda imitar. Esta es la razón para hablar del valor de la estrategia de educar con el ejemplo.

Además, los terapeutas ocupacionales tienen en sus manos la posibilidad de apoyar y conducir al niño en la comprensión y aceptación de principios y valores para vivir en y para su comunidad (Restrepo, 1992; Trujillo, 2002). De esta manera la interacción del terapeuta se hará en el ambiente natural del niño con el fin de enseñarle la importancia de sus acciones para trasformar la realidad que vive con base en una escala de valores que promueva la equidad y justicia social.

Además los maestros y terapeutas que actúan como educadores son los facilitadores de las actuaciones democráticas del grupo cuando enseñan la concertación, la negociación y la creación de valores como la igualdad, la solidaridad o la justicia. Todo esto será la base de actuaciones que buscan el bien común y la participación comunitaria.

También es tarea del maestro y del terapeuta ocupacional favorecer las condiciones para que el niño empiece a desarrollar la autonomía la cual será el punto de partida para la construcción de valores y el desarrollo de una postura ética. A continuación se encuentra un cuadro que muestra ejemplos de las actitudes que se pueden utilizar para favorecer la formación en valores. 


\begin{tabular}{|c|c|}
\hline $\begin{array}{l}\text { EL MAESTRO O TERAPEUTA } \\
\text { PUEDEN ACTUAR }\end{array}$ & PARA FAVORECER EN EL NIN̄O \\
\hline Enseñando con el ejemplo & Patrones apropiados de acción. La imitación de conductas asertivas. \\
\hline Demostrando una actitud de ayuda & La actitud de entrega y servicio desde una postura de ternura. \\
\hline Animando a la independencia & $\begin{array}{l}\text { La seguridad y autonomia para que pueda decidir por si mismo. } \\
\text { Escoger y decidir lo que quiere hacer. }\end{array}$ \\
\hline Conociendo al niño & La seguridad y la autoimagen. El desarrollo de la autoestima. \\
\hline Creyendo en el niño & $\begin{array}{l}\text { Sentimiento de seguridad y confianza. Reconocer que se pue- } \\
\text { de equivocar y puede rectificar. Creer en si mismo y en los } \\
\text { valores que empieza a desarrollar y practicar. }\end{array}$ \\
\hline Favoreciendo la autoestima & $\begin{array}{l}\text { El autoconcepto positivo. El sentido de capacidad. La tolerancia y } \\
\text { aceptación del otro. El respeto a la vida. La aceptación personal. }\end{array}$ \\
\hline $\begin{array}{l}\text { Sabiendo cómo interesar al niño en } \\
\text { los temas sobre valores }\end{array}$ & La exploración, análisis y construcción de saberes. \\
\hline $\begin{array}{l}\text { Aceptando las diferencias entre los } \\
\text { niños }\end{array}$ & $\begin{array}{l}\text { El desarrollo de la individualidad, la capacidad de escoger. } \\
\text { Actuar con independencia pero respetando los espacios de los } \\
\text { demás. Valorar la diversidad. Encontrar la riqueza en la diferen- } \\
\text { cia. Aceptar las diversas formas de pensar y actuar. Aprender a } \\
\text { negociar, conciliar y concertar. }\end{array}$ \\
\hline $\begin{array}{l}\text { Respetando su manera de ser y sus } \\
\text { derechos }\end{array}$ & $\begin{array}{l}\text { La aceptación y el reconocimiento de derechos. Identificando } \\
\text { sus necesidades, debilidades y fortalezas. }\end{array}$ \\
\hline $\begin{array}{l}\text { Adaptándose a lo que el niño nece- } \\
\text { sita }\end{array}$ & $\begin{array}{l}\text { La capacidad de respetar el ritmo de los demás. La tolerancia y } \\
\text { el respeto a la diferencia. }\end{array}$ \\
\hline $\begin{array}{l}\text { Animando al niño a actuar con segu- } \\
\text { ridad }\end{array}$ & $\begin{array}{l}\text { Una actitud amorosa y tierna que permita el encuentro y que } \\
\text { motive a actuar de manera autónoma y segura. } \\
\text { Logrando desarrollar los valores de ayuda y entrega }\end{array}$ \\
\hline $\begin{array}{l}\text { Creando un sentido de pertenencia } \\
\text { a su comunidad y cultura }\end{array}$ & $\begin{array}{l}\text { La seguridad, y el sentido de comunidad. Se favorece la iden- } \\
\text { tificación al grupo y el trabajo en equipo. }\end{array}$ \\
\hline $\begin{array}{l}\text { Mostrando alegria y esperanza en el } \\
\text { pais, en el futuro }\end{array}$ & $\begin{array}{l}\text { El entusiasmo y el optimismo. Para dar importancia a la alegria } \\
\text { y enseñar el amor por lo que se hace. Para que aprenda a creer } \\
\text { en el otro. }\end{array}$ \\
\hline Siendo justo en cada situación & $\begin{array}{l}\text { La importancia del trabajo equitativo. El valor de la justicia. } \\
\text { Desarrollar la confianza para resolver algunos de sus problemas } \\
\text { y pedir ayuda para aquellos que no puede. }\end{array}$ \\
\hline Teniendo expectactivas alcanzables & $\begin{array}{l}\text { La sensación de capacidad. El sentido de libertad de acción. La } \\
\text { responsabilidad por lo que se es capaz de hacer. La construc- } \\
\text { ción de las propias normas. }\end{array}$ \\
\hline $\begin{array}{l}\text { Formando el carácter del niño hacia } \\
\text { una postura ética }\end{array}$ & $\begin{array}{l}\text { El logro de la franqueza, la justicia y la verdad como producto de } \\
\text { las oportunidades Una actitud ética. La capacidad de resolver los } \\
\text { problemas cotidianos. Iniciar la capacidad de resolver los conflic- } \\
\text { tos a través del dialogo, la negociación o la reconciliación. }\end{array}$ \\
\hline $\begin{array}{l}\text { Enseñando al niño a actuar demo- } \\
\text { cráticamente }\end{array}$ & $\begin{array}{l}\text { El sentido de ciudadano responsable y productivo. El conocimien- } \\
\text { to de deberes y derechos. La importancia del servicio y el trabajo } \\
\text { en equipo. El valor del bien común. Ensayar las estrategias para } \\
\text { dar opiniones y proponer reglas de convivencia grupal Practicar la } \\
\text { autoreflexión para entender las situaciones y darles solución. }\end{array}$ \\
\hline $\begin{array}{l}\text { Promoviendo la participación social } \\
\text { del niño en su propia comunidad }\end{array}$ & $\begin{array}{l}\text { Autonomia y madurez para actuar. Capacidad para resolver } \\
\text { problemas, defender y exponer ideas, reconocer y rectificar erro- } \\
\text { res, expresar sentimientos y respetar a los demás. }\end{array}$ \\
\hline
\end{tabular}

Fuente: Restrepo y colaboradoras (5); Amar y Amar (6); Pinto (7); Sauri (8).

Cuadro No. 2. Actitudes de los educadores para favorecer en el niño la formación en valores. 
Para resumir se puede decir que los niños siempre están observando a los adultos para aprender sobre la forma de interactuar con los demás, resolver conflictos, plantear problemas o expresar opiniones. Por éste motivo tanto los maestros como los terapeu- tas y demás adultos del entorno escolar deben estar conscientes de sus actitudes. Estas serán más importantes que lo que digan ya que el niño dará mayor valor a las acciones y al ejemplo que recibe que a lo que le digan o aconsejen que haga.

\section{* Programa de formación de valores del jardín infantil por un mañana, un ejemplo de la implementación de la propuesta teórica aquí planteada}

La Formación en valores de los niños en edad preescolar forma parte del programa de Desarrollo Emocional del jardín Infantil Por un Mañana el cual es uno de los pilares del proyecto educativo institucional (9).

El equipo que lo diseñó y dirige en la actualidad cuenta con la participación de una terapeuta ocupacional quien promueve el desarrollo de la comunidad educativa (padres, niños y maestros) con el empleo de estrategias que motivan al crecimiento ético de las personas.

A través de su intervención la terapeuta ocupacional también busca que cuando los adultos y los niños realicen sus ocupaciones logren la satisfacción personal teniendo como requisito el desarrollo de una escala valorativa adecuada la cual les permi- ta realizar actuaciones democráticas y acciones en pro del bien comunitario (Trujillo, 2002).

Este programa tiene sus bases teóricas en los planteamientos de Daniel Goleman sobre Inteligencia Emocional; Luis Carlos Restrepo sobre el derecho a la ternura; Martha Rodríguez sobre escuela y actitud democrática; Lucia Pinto sobre servicio social y postura ética y Kielhofner sobre la importancia del sistema de la voluntad en el desarrollo ético.

El programa de desarrollo emocional ofrece estrategias concretas para favorecer la formación de valores en los niños preescolares. Algunas de estas estrategias se han recopilado en la Cartilla de Desarrollo Emocional (10). El cuadro que se encuentra a continuación resume esas estrategias. 


\section{ESTRATEGIAS DEL PROGRAMA DE DESARROLLO EMOCIONAL QUE PROMUEVEN LA FORMACIÓN EN VALORES}

El conocimiento de las capacidades personales para que los niños desarrollen la autoestima.

La aceptación de fortalezas y debilidades tanto en sí mismos como el los demás.

El empleo del ejemplo para favorecer la forma correcta de expresarse, crear una autoimagen positiva e iniciar al niño en la construcción de los determinantes valorativos.

El desarrollo sensoriomotor y la expresión corporal creativa para expresar sentimientos y estados de ánimo de manera adecuada.

El moldeo de actitudes de autoregulación y expresión.

El aprendizaje de los derechos de los niños y los deberes que estos conllevan.

Desarrollar del pensamiento infantil por medio de la interpretación de situaciones, análisis de puntos de vista y adecuación de maneras de expresión entre los niños.

El desarrollo de actitudes éticas como el respeto, la solidaridad, la justicia o la participación

Facilitar la comprensión de cada valor, al hacer de interlocutores que le den puntos de vista, opciones y argumentos de juicio a los compañeros.

El reconocimiento de la existencia del otro y del sentido de grupo a partir del respeto de los derechos y deberes que se tienen para vivir en comunidad.

La necesidad de resolver los conflictos de manera pacífica.

La importancia de cuidar el bien común.

El aprendizaje de los principios éticos que son aceptados en su contexto cultural.

La importancia de actuar de acuerdo con normas de convivencia escogidas y aceptadas por el grupo.

Facilitar las reflexiones del niño para analizar situaciones, buscar soluciones y decidir cómo actuar.

La importancia de ser parte activa de la comunidad para promover el desarrollo de la misma.

La participación en actividades comunitarias que favorecen la capacidad de actuar democráticamente.

La necesidad de crecer con una actitud solidaria hacia los que necesitan ayuda.

La convicción de que se puede cambiar el entorno a partir de acciones concretas que permitan el bienestar de la comunidad en que se vive.

El amor por las manifestaciones culturales y sociales de su comunidad.

El sentido de patria y el amor por su pais.

Fuente Amaya de Lee y colaboradoras (9).

Cuadro No. 3. Estrategias del programa de desarrollo emocional que promueven la formación en valores.

El programa también cuenta con un conjunto de talleres, lecturas para reflexionar y planes caseros con el fin de involucrar a la familia en la tarea de ayudar al niño en la construcción de valores y principios éticos en su comunidad.
Con el programa de Desarrollo Emocional se ha logrado crear conciencia en todos los adultos que participan de la comunidad escolar -padres, maestros y terapeutas de su importante papel en la formación de valores en los niños en edad preescolar. 
Con frecuencia se reciben testimonios de las familias que han visto cómo sus niños se destacan dentro de su comunidad al demostrar el desarrollo del área moral y ética con sus actitudes y comentarios.

A manera de conclusión se puede decir que la misión de los maestros y terapeutas ocupacionales que participan de los programas de formación en valores es favorecer el desarrollo ético de los niños a partir de propuestas pedagógicas que integren todas las dimensiones del desarrollo infantil. La importancia de este programa se puede ejemplificar con las palabras del educador colombiano Agustín Nieto Caballero (10):

"Ya no se trata únicamente de preparar profesionales. Un propósito de información intelectual y moral mueve la acción de todos los educadores. Se trata de iluminar la conciencia, de forjar la personalidad, de acrecentar las energías, de poner en primer término el sentimiento de la responsabilidad, de adaptar el individuo a las nuevas circunstancias, de darle las disciplinas necesarias para que pueda vencer las resistencias que se le presenten, de cultivar en la gente moza la sensibilidad social". 
1. Revista de la comisión internacional de juristas. Convención sobre los derechos del niño. Número 44.1990.

2. Rodríguez, Martha. La escuela: el primer espacio de actuación pública del niño. Criterios y observaciones en la formación de hábitos ciudadanos. Bogotá, Colombia. Folleto de la Fundación Social. 1997.

3. Unete a la fuerza de la paz. Folleto de movimiento voto por la paz. País libre y otros. Bogotá, Colombia. 1999.

4. Ministerio de Educación Nacional. Preescolar, lineamientos pedagógicos. Serie lineamientos curriculares. Bogotá, Colombia Creamos Alternativas. 1998.

5. Restrepo de Franco, Beatriz. Mejía, Y. Tobón, M. Robles, D. Rodríguez, P. Trujillo de Prías, C. Lizarazo, R. Amaya de Lee, M. Construcción de valores en el niño durante la vida preescolar. Trabajo de grado para obtener el ingreso al Escalafón Docente. Bogotá, CoIombia. DIE, CEP. 1992.

6. Amar Amar, José Juan. La participación de los niños. En: Espacio para la infancia. Revista periódica Bernard Van Leer Foundation. No. 14, Julio de 2000.

7. Pinto, Lucía. La formación de la afectividad. Programa Escuela de padres. Santafé de Bogotá. Federación de asociaciones de padres de los colegios de la compañía de Jesús. 1990.

8. Sauri Suárez, Gerardo. Participación infantil: Herramienta educativa y de desarrollo. En: Espacio para la infancia. No. 14, Julio de 2000. Revista periódica Bernard Van Leer Foundation.

9. Amaya de Lee, Mariluces. B. Restrepo de Franco. C. E. De Prías. A. R. Lizarazo. M Tobón de Awad. Programa para el desarrollo emocional de los niños preescolares. Revista Ocupación Humana. 1999 Vol. 8 No. 1.

10. Nieto Caballero, Agustín. Escuela Activa. Bogotá, Colombia Ed. Presencia, Gimnasio Moderno. 19987

11. Barquer Schwarts, Kathleen. Occupational therapy and education: a Shared vision. The American journal of Occupational Therapy. 1992 Vol. 46 No. 1.

12. Chen, Jen-suh. G. Kielhofner. C. De Las Heras. L. Magalhaes. The volitional questionnaire: psichometric development and practical use. The American Journal of Occupational Therapy. 1996 July - August, Vol. 50 No. 7.

13. Engquist, Douglas. M. Short-De graff. J. Gliner, K. Oltjenbruns. Occupational therapis beliefs and practices with regard to spirituality and therapy. The American Journal of Occupational Therapy. 1997
March Vol 51, No. 3.

14. Goleman, Daniel La inteligencia emocional. Nueva Cork (USA). Bantam, Editors. New York. 1995.

15. Iceta, Manuel. Dejar ser, educar desde el encuentro personal. Madrid, (España) Ediciones S. M. 1985.

16. Hopkins, Helen. Fundamentos teóricos y filosóficos actuales de la Terapia Ocupacional. En: Willard and Spackman. Occupational Therapy. Philadelphia. USA. Lippincott Editors. 1998 P, 58 - 59.

17. Howard, Brenda. J. Howard. Occupation as spiritual activity. The American Journal of Occupational Therapy. 1997 March Vol 51, No. 3.

18. Kielhofner, Gary. The emerging paradigm of Ocupational Therapy. En: Conceptual fundations of Occupational Therapy. F. A. Davis Company. Philadelphia. USA 1992.

19. Labinowicz, Ed. Introducción a Piaget. Pensamiento, aprendizaje, enseñanza. Wilmington, Del (USA). Fondo Educativo Interamericano. 1982.

20. Medina, Soraya. Derechos a mi medida. Ciudadanos desde el preescolar. En: Espacio para la infancia. Revista periódica Bernard Van Leer Foundation. No. 14, Julio de 2000.

21. Ministerio de Educación Nacional . La paz de los mil días. Actos de paz desde mi colegio. Bogotá, Colombia. Zeta periodismo. 1997.

22. Ortiz de Maschwitz, Helena. Inteligencias múltiples en la educación de la persona. Bogotá, Colombia. Editorial Nomos. 2000.

23. Restrepo, Luis Carlos. Ecología humana: Una propuesta de reconstrucción cultural desde la ternura. Pontifica Universidad Javeriana. Facultad de Psicología, Programa de Educación continuada. Bogotá, Colombia. 1994.

24. El derecho a la ternura. Bogotá, Colombia. Arango Editores. 1994.

25. Tobón, Martha. Restrepo de Franco, B. Lizarazo, R. Trujillo de Prías, C. Amaya de Lee, M. Cartilla de desarrollo emocional para los niños preescolares. Bogotá, Colombia Ed: Jardín Infantil Por un Mañana. 1999.

26. Trujillo, Alicia. Terapia Ocupacional: Definición, naturaleza, misión, propósito, procedimientos y campos de acción. En: Terapia Ocupacional. Conocimiento y práctica en Colombia. Colección Sede. Bogotá, Colombia. Universidad Nacional de Colombia. 2002.

27. Vigotski, Lev. Pensamiento y lenguaje. Buenos Aires (Argentina). Editorial La Pléyade. 1981. 